\title{
On an open problem about a class of optimal ternary cyclic codes
}

\author{
Dongchun Han ${ }^{\mathrm{a}}$, Haode Yan $^{\mathrm{a}, *}$ \\ ${ }^{a}$ School of Mathematics, Southwest Jiaotong University, Chengdu, 610031, China
}

\begin{abstract}
Cyclic codes are a subclass of linear codes and have applications in consumer electronics, data storage systems and communication systems as they have efficient encoding and decoding algorithms. In this paper, we settle an open problem about a class of optimal ternary cyclic codes which was proposed by Ding and Helleseth [6]. Let $\mathcal{C}_{(1, e)}$ be a cyclic code of length $3^{m}-1$ over $\mathbb{G} \mathbb{F}(3)$ with two nonzeros $\alpha$ and $\alpha^{e}$, where $\alpha$ is a generator of $\mathbb{G} \mathbb{F}\left(3^{m}\right)^{*}$ and $e$ is a given integer. It is shown that $\mathcal{C}_{(1, e)}$ is optimal with parameters $\left[3^{m}-1,3^{m}-1-2 m, 4\right]$ if one of the following conditions is met. 1) $m \equiv 0(\bmod 4), m \geq 4$, and $e=3^{\frac{m}{2}}+5$. 2) $m \equiv 2(\bmod 4), m \geq 6$, and $e=3^{\frac{m+2}{2}}+5$.
\end{abstract}

Keywords:

Cyclic code, optimal code, ternary code, Sphere Packing bound.

2000 MSC: 94B15, $11 \mathrm{~T} 71$

\section{Introduction}

Cyclic codes are an important subclass of linear codes and have been extensively studied [15]. Let $p$ be a prime, $m$ be a positive integer. Let $\mathbb{G} \mathbb{F}(p)$ and $\mathbb{G} \mathbb{F}\left(p^{m}\right)$ denote the finite fields with $p$ and $p^{m}$ elements, respectively. A linear $[n, k, d]$ code $C$ over the finite field $\mathbb{G F}(p)$ is a $k$-dimensional subspace of $\mathbb{G} \mathbb{F}(p)^{n}$ with minimum Hamming distance $d$, and is called cyclic if any cyclic shift of a codeword is another codeword of $\mathcal{C}$. Let $\operatorname{gcd}(n, p)=1$. By identifying any vector $\left(c_{0}, c_{1}, \cdots, c_{n-1}\right) \in \mathbb{G F}(p)^{n}$ with

$$
c_{0}+c_{1} x+c_{2} x^{2}+\cdots+c_{n-1} x^{n-1} \in \mathbb{G} \mathbb{F}(p)[x] /\left(x^{n}-1\right),
$$

any cyclic code of length $n$ over $\mathbb{G} \mathbb{F}(p)$ corresponds to an ideal of the polynomial residue class ring $\mathbb{G} \mathbb{F}(p)[x] /\left(x^{n}-1\right)$. It is well known that every ideal of $\mathbb{G} \mathbb{F}(p)[x] /\left(x^{n}-1\right)$ is principal. Any cyclic code $\mathcal{C}$ can be expressed as $\mathcal{C}=\langle g(x)\rangle$, where $g(x)$ is monic and has the least degree. Then $g(x)$ is called the generator polynomial and $h(x)=\left(x^{n}-1\right) / g(x)$ is referred to as the parity-check

\footnotetext{
${ }^{2}$ D. Han's research was supported by the National Science Foundation of China Grant No.11601448 and the Fundamental Research Funds for the Central Universities of China under Grant 2682016CX121. H. Yan's research was supported by the National Natural Science Foundation of China Grant No.11801468 and the Fundamental Research Funds for the Central Universities of China under Grant 2682018 CX61.

* Corresponding author

Email addresses: han-qingfeng@163.com (Dongchun Han), hdyan@swjtu.edu.cn (Haode Yan)
} 
polynomial of $\mathcal{C}$. For some recent developments of cyclic codes, the readers are referred to [1], [3]-[6], [10], [12], [16]-[19], [22]-[25], [27]-[30] and the references therein.

Let $\alpha$ be a generator of $\mathbb{G F}\left(3^{m}\right)^{*}=\mathbb{G F}\left(3^{m}\right) \backslash\{0\}$ and $m_{i}(x)$ be the minimal polynomial of $\alpha^{i}$ over $\mathbb{G F}(3)$, where $1 \leq i \leq 3^{m}-1$. Let $C_{(1, e)}$ be the cyclic code over $\mathbb{G F}(3)$ with generator polynomial $m_{1}(x) m_{e}(x)$, where $e$ is an integer such that $\alpha$ and $\alpha^{e}$ are nonconjugate. Carlet, Ding and Yuan [1] proved that $\mathcal{C}_{(1, e)}$ has parameters $\left[3^{m}-1,3^{m}-1-2 m, 4\right]$ when $x^{e}$ are certain perfect nonlinear monomials over $\mathbb{G F}\left(3^{m}\right)$. Notice that the ternary cyclic code with parameters $\left[3^{m}-1,3^{m}-1-2 m, 4\right]$ is optimal according to the Sphere Packing bound. In 2013, Ding and Helleseth [6] constructed several classes of optimal ternary cyclic codes $\mathcal{C}_{(1, e)}$ with parameters $\left[3^{m}-1,3^{m}-1-2 m, 4\right]$ by employing some monomials $x^{e}$ over $\mathbb{G F}\left(3^{m}\right)$ including almost perfect nonlinear monomials. In addition, nine open problems about $\mathcal{C}_{(1, e)}$ with parameters $\left[3^{m}-1,3^{m}-\right.$ $1-2 m, 4]$ were proposed in [6]. Recently, two of the nine open problems were solved, see [19, 20]. Moreover, an open problem proposed in [6] is shown as follows.

Open Problem 1.1 (Open Problem 7.12, [6]). Let $e=3^{h}+5$, where $2 \leq h \leq m-1$. Let $m$ be even. Is it true that the ternary cyclic code $\mathcal{C}_{(1, e)}$ has parameters $\left[3^{m}-1,3^{m}-1-2 m, 4\right]$ if one of the following conditions is met?

1. $m \equiv 0(\bmod 4), m \geq 4$, and $h=\frac{m}{2}$.

2. $m \equiv 2(\bmod 4), m \geq 6$, and $h=\frac{m+2}{2}$.

In this paper, we will settle this open problem. The rest of this paper is organized as follows. In section 2 we introduce two useful results which will be employed in the sequel. In Section, 3 , we present the proof of our main result. Section 4 concludes the paper with some remarks.

\section{Preliminaries}

In this section, we will introduce two useful results. The first one is about the cyclotomic coset. For a prime $p$, the $p$-cyclotomic coset modulo $p^{m}-1$ containing $j$ is defined as

$$
C_{j}=\left\{j p^{s} \bmod \left(p^{m}-1\right): s=0,1, \ldots, m-1\right\} .
$$

We have the following lemma.

Lemma 2.1 (Lemma 2.1, [6]). For any $1 \leq e \leq p^{m}-2$ with $\operatorname{gcd}\left(e, p^{m}-1\right)=2$, the cardinality of the p-cyclotomic coset $C_{e}$ is equal to $m$.

It is known that a code with parameters $\left[3^{m}-1,3^{m}-1-2 m, 4\right]$ is optimal according to the Sphere Packing bound. To determine the optimality of $\mathcal{C}_{(1, e)}$, the following sufficient and necessary conditions are given by Ding and Helleseth in [6].

Theorem 2.2 (Theorem 4.1, [6]). Let $e \notin \mathcal{C}_{1}$, and $\left|\mathcal{C}_{e}\right|=m$. The ternary cyclic code $\mathcal{C}_{(1, e)}$ has parameters $\left[3^{m}-1,3^{m}-1-2 m, 4\right]$ if and only if the following conditions are satisfied:

Cl: $e$ is even;

C2: the equation $(x+1)^{e}-x^{e}-1=0$ has the only solution $x=0$ in $\mathbb{G F}\left(3^{m}\right)$;

C3: the equation $(x+1)^{e}+x^{e}+1=0$ has the only solution $x=1$ in $\mathbb{G F}\left(3^{m}\right)$. 


\section{Solving Open Problem 1.1}

In this section, we confirm that each condition in Open Problem 1.1 satisfies all the three conditions in Theorem 2.2. Then the answer of the open problem can be deduced. Firstly, we confirm that $\mathrm{C} 1$ holds in the following lemma.

Lemma 3.1. Let $e=3^{h}+5$, where $2 \leq h \leq m-1$. Then $e \notin \mathcal{C}_{1}$ and $\left|\mathcal{C}_{e}\right|=m$ if one of the following conditions is met.

1. $m \equiv 0(\bmod 4), m \geq 4$, and $h=\frac{m}{2}$.

2. $m \equiv 2(\bmod 4), m \geq 6$, and $h=\frac{m+2}{2}$.

Proof. We only prove the first one and the second one is similar. It is easy to see that $e \notin \mathcal{C}_{1}$ since $e$ is even. It will be shown that $\left|\mathcal{C}_{e}\right|=m$. We have

$$
\begin{aligned}
\operatorname{gcd}\left(e, 3^{m}-1\right) & =\operatorname{gcd}\left(3^{\frac{m}{2}}+5,3^{m}-1\right)=\operatorname{gcd}\left(3^{\frac{m}{2}}+5,3^{m}-1-\left(3^{\frac{m}{2}}+5\right)\left(3^{\frac{m}{2}}-5\right)\right) \\
& =\operatorname{gcd}\left(3^{\frac{m}{2}}+5,24\right)=\operatorname{gcd}\left(3^{\frac{m}{2}}+5,8\right)=(6,8)=2 .
\end{aligned}
$$

The fifth equality holds since $m \equiv 0(\bmod 4)$ and $3^{\frac{m}{2}}+5 \equiv 6(\bmod 8)$. Consequently, $\left|\mathcal{C}_{e}\right|=m$ follows from lemma 2.1 .

Secondly, we investigate the solutions of $(x+1)^{e}-x^{e}-1=0$ in $\mathbb{G F}\left(3^{m}\right)$.

Lemma 3.2. Let $e=3^{h}+5$, where $2 \leq h \leq m-1$. Then

$$
(x+1)^{e}-x^{e}-1=0
$$

has the only solution $x=0$ in $\mathbb{G F}\left(3^{m}\right)$ if one of the following conditions is met.

1. $m \equiv 0(\bmod 4), m \geq 4$, and $h=\frac{m}{2}$.

2. $m \equiv 2(\bmod 4), m \geq 6$, and $h=\frac{m+2}{2}$.

Proof. It is obvious that $x=0$ is a solution of (1) and $x= \pm 1$ is not. Suppose that $\theta \in \mathbb{G F}\left(3^{m}\right) \backslash$ $\mathbb{G F}(3)$ and is a solution of (1). Through a straight calculation, we have that

$$
\theta^{3^{h}-1}\left(\theta^{4}-\theta^{3}-\theta^{2}+\theta-1\right)=\theta^{4}-\theta^{3}+\theta^{2}+\theta-1 .
$$

First, we assert that $\theta^{4}-\theta^{3}-\theta^{2}+\theta-1 \neq 0$. Otherwise, we have $\theta^{4}-\theta^{3}-\theta^{2}+\theta-1=\theta^{4}-$ $\theta^{3}+\theta^{2}+\theta-1=0$, which leads to $\theta=0$. It is a contradiction. Hence we have

$$
\theta^{3^{h}}=\frac{f(\theta)}{g(\theta)}
$$

where $f(\theta)=\theta^{5}-\theta^{4}+\theta^{3}+\theta^{2}-\theta$ and $g(\theta)=\theta^{4}-\theta^{3}-\theta^{2}+\theta-1$. Taking $3^{h}$ powers on both sides of the equation (2), we have

$$
\theta^{3^{2 h}}=\frac{\theta^{5 \cdot 3^{h}}-\theta^{4 \cdot 3^{h}}+\theta^{3 \cdot 3^{h}}+\theta^{2 \cdot 3^{h}}-\theta^{3^{h}}}{\theta^{4 \cdot 3^{h}}-\theta^{3 \cdot 3^{h}}-\theta^{2 \cdot 3^{h}}+\theta^{\cdot 3^{h}}-1}
$$

Plugging (2) into $[3]$, we obtain

$$
\theta^{3^{2 h}}=\frac{F(\theta)}{G(\theta)},
$$


where $F(\theta)=f(\theta)^{5}-f(\theta)^{4} g(\theta)+f(\theta)^{3} g(\theta)^{2}+f(\theta)^{2} g(\theta)^{3}-f(\theta) g(\theta)^{4}$ and $G(\theta)=f(\theta)^{4} g(\theta)-$ $f(\theta)^{3} g(\theta)^{2}-f(\theta)^{2} g(\theta)^{3}+f(\theta) g(\theta)^{4}-g(\theta)^{5}$. We distinguish the following two cases.

Case $1: m \equiv 0(\bmod 4), m \geq 4$, and $h=\frac{m}{2}$.

Noting that $\theta^{32 h}=\theta$ since $2 h=m$, then (4) becomes

$$
\theta G(\theta)-F(\theta)=0 .
$$

With the help of Magam Program, we can decompose the left-hand side of the above equation into the product of some irreducible factors as follows.

$$
\theta^{3}(\theta+1)(\theta-1)\left(\theta^{6}+\theta^{3}-\theta+1\right)\left(\theta^{6}-\theta^{5}+\theta^{3}+1\right)\left(\theta^{6}-\theta^{5}-\theta^{3}-\theta+1\right)=0 .
$$

If $\theta^{6}+\theta^{3}-\theta+1=0$, then $\theta \in \mathbb{G} \mathbb{F}\left(3^{6}\right) \subseteq \mathbb{G} \mathbb{F}\left(3^{m}\right)$. We have $6 \mid m$ and then $6 \mid h$ since $m=2 h$ and $h$ is even. Plugging $\theta^{3^{h}}=\theta$ into the equation (2), we have

$$
\theta=\frac{\theta^{5}-\theta^{4}+\theta^{3}+\theta^{2}-\theta}{\theta^{4}-\theta^{3}-\theta^{2}+\theta-1}
$$

which leads to $\theta=0$. It is a contradiction. Similarly, we can prove that $\theta^{6}-\theta^{5}+\theta^{3}+1 \neq 0$ and $\theta^{6}-\theta^{5}-\theta^{3}-\theta+1 \neq 0$. Then $x=0$ is the only solution of $(1)$ in $\mathbb{G F}\left(3^{m}\right)$.

Case 2: $m \equiv 2(\bmod 4), m \geq 6$, and $h=\frac{m+2}{2}$.

Noting that $\theta^{3^{2 h}}=\theta^{9}$ since $2 h=m+2$, then (4) becomes

$$
\theta^{9} G(\theta)-F(\theta)=0 .
$$

With the help of Magam Program, we can decompose the left-hand side of the above equation into the product of some irreducible factors as follows.

$$
\begin{gathered}
\theta(\theta+1)(\theta-1)\left(\theta^{4}+\theta^{3}-\theta^{2}-\theta-1\right)\left(\theta^{4}+\theta^{3}+\theta^{2}-\theta-1\right)\left(\theta^{6}-\theta^{5}+\theta^{4}-\theta^{3}+\theta^{2}-\theta+1\right) \\
\left(\theta^{8}+\theta^{7}+\theta^{6}-\theta^{4}+\theta^{2}+\theta+1\right)\left(\theta^{8}+\theta^{7}-\theta^{6}-\theta^{2}+\theta+1\right)=0 .
\end{gathered}
$$

If $\theta^{6}-\theta^{5}+\theta^{4}-\theta^{3}+\theta^{2}-\theta+1=0$, then $\theta \in \mathbb{G F}\left(3^{6}\right) \subseteq \mathbb{G F}\left(3^{m}\right)$ and $6 \mid m$. It follows from $h$ is even that $h \equiv 4(\bmod 6)$. Noting that $\theta^{7}=-1$, we obtain $\theta^{3^{h}}=\theta^{3^{4}}=\theta^{77+4}=-\theta^{4}$. Plugging this into (2), we obtain

$$
\theta^{6}+\theta^{5}+\theta^{4}-\theta^{3}-\theta^{2}-\theta-1=0 .
$$

This together with $\theta^{6}-\theta^{5}+\theta^{4}-\theta^{3}+\theta^{2}-\theta+1=0$ leads to $\theta^{5}-\theta^{2}-1=\theta^{5}+\theta^{3}-\theta^{2}-1=0$. Then $\theta$ must be zero, which is a contradiction. Moreover, $\theta^{4}+\theta^{3}-\theta^{2}-\theta-1, \theta^{4}+\theta^{3}+\theta^{2}-$ $\theta-1, \theta^{8}+\theta^{7}+\theta^{6}-\theta^{4}+\theta^{2}+\theta+1$ and $\theta^{8}+\theta^{7}-\theta^{6}-\theta^{2}+\theta+1$ cannot be zero since $4 \nmid m$. Then $x=0$ is the only solution of $(1)$ in $\mathbb{G F}\left(3^{m}\right)$. This completes the proof.

In what follows, we investigate the solutions of $(x+1)^{e}+x^{e}+1=0$ in $\mathbb{G F}\left(3^{m}\right)$.

Lemma 3.3. Let $e=3^{h}+5$, where $2 \leq h \leq m-1$. Then

$$
(x+1)^{e}+x^{e}+1=0
$$

has the only solution $x=1$ in $\mathbb{G F}\left(3^{m}\right)$ if one of the following conditions is met.

1. $m \equiv 0(\bmod 4), m \geq 4$, and $h=\frac{m}{2}$. 
2. $m \equiv 2(\bmod 4), m \geq 6$, and $h=\frac{m+2}{2}$.

Proof. It is obvious that $x=1$ is a solution of (5). Suppose that $\theta \in \mathbb{G F}\left(3^{m}\right) \backslash \mathbb{G F}(3)$ is a solution of (5). Through a straight calculation, we have

$$
\theta^{3^{h}}\left(\theta^{4}-\theta^{3}+\theta^{2}+1\right)=\theta^{4}+\theta^{2}-\theta+1 .
$$

First, we assert that $\theta^{4}-\theta^{3}+\theta^{2}+1 \neq 0$. Otherwise, we have $\theta^{4}-\theta^{3}+\theta^{2}+1=\theta^{4}+\theta^{2}-\theta+1=$ 0 . It then follows that $\theta^{3}=\theta$. This is contrary to the assumption that $\theta \notin \mathbb{G F}(3)$. Hence, we have

$$
\theta^{3^{h}}=\frac{k(\theta)}{l(\theta)}
$$

where $k(\theta)=\theta^{4}+\theta^{2}-\theta+1$ and $l(\theta)=\theta^{4}-\theta^{3}+\theta^{2}+1$. Taking $3^{h}$ powers on both sides of the equation (6), we have

$$
\theta^{3^{2 h}}=\frac{\theta^{4 \cdot 3^{h}}+\theta^{2 \cdot 3^{h}}-\theta^{3^{h}}+1}{\theta^{4 \cdot 3^{h}}-\theta^{3 \cdot 3^{h}}+\theta^{2 \cdot 3^{h}}+1}
$$

Plugging (6) into (7), we obtain

$$
\theta^{3^{2 h}}=\frac{K(\theta)}{L(\theta)},
$$

where $K(\theta)=k(\theta)^{4}+k(\theta)^{2} l(\theta)^{2}-k(\theta) l(\theta)^{3}+l(\theta)^{4}$ and $L(\theta)=k(\theta)^{4}-k(\theta)^{3} l(\theta)+k(\theta)^{2} l(\theta)^{2}+$ $l(\theta)^{4}$. We distinguish the following two cases.

Case $1: m \equiv 0(\bmod 4), m \geq 4$, and $h=\frac{m}{2}$.

Noting that $\theta^{3^{2 h}}=\theta$ since $2 h=m$, then $\theta$ satisfies

$$
\theta L(\theta)-K(\theta)=0
$$

With the help of Magam Program, we can decompose the left-hand side of the above equation into the product of some irreducible factors as follows.

$$
(\theta-1)^{5}\left(\theta^{2}+\theta-1\right)^{2}\left(\theta^{2}-\theta-1\right)^{2}\left(\theta^{2}+1\right)^{2}=0 .
$$

If $\theta^{2}+\theta-1=0$, then $\theta \in \mathbb{G F}\left(3^{2}\right)$. We have $\theta^{3^{2}}=\theta$ and then $\theta^{3^{h}}=\theta$ since $h$ is even. Plugging $\theta^{3^{h}}=\theta$ into (6), then we have

$$
\theta=\frac{\theta^{4}+\theta^{2}-\theta+1}{\theta^{4}-\theta^{3}+\theta^{2}+1}
$$

which leads to $\theta^{5}+\theta^{4}+\theta^{3}-\theta^{2}-\theta-1=(\theta-1)^{5}=0$, a contradiction. Similarly, we can prove that $\theta^{2}+\theta-1 \neq 0$ and $\theta^{2}+1 \neq 0$. Then $x=1$ is the only solution of $(5)$ in $\mathbb{G F}\left(3^{m}\right)$.

Case 2: $m \equiv 2(\bmod 4), m \geq 6$, and $h=\frac{m+2}{2}$.

Noting that $\theta^{3^{2 h}}=\theta^{9}$ since $2 h=m+2$, then $\theta$ satisfies

$$
\theta^{9} L(\theta)-K(\theta)=0 .
$$

With the help of Magam Program, we can decompose the left-hand side of the above equation into the product of some irreducible factors as follows.

$$
(\theta-1)\left(\theta^{2}+1\right)\left(\theta^{2}+\theta-1\right)\left(\theta^{2}-\theta-1\right)\left(\theta^{3}-\theta+1\right)\left(\theta^{3}-\theta-1\right)
$$




$$
\left(\theta^{3}+\theta^{2}-\theta+1\right)\left(\theta^{3}-\theta^{2}+\theta+1\right)\left(\theta^{3}+\theta^{2}-1\right)\left(\theta^{3}-\theta^{2}+1\right)=0 .
$$

Similar with the proof of Case 1 , we know that $\theta^{2}+1, \theta^{2}+\theta-1, \theta^{2}-\theta-1 \neq 0$. If $\theta^{3}-\theta-1=0$, then $\theta \in \mathbb{G F}\left(3^{3}\right) \subseteq \mathbb{G F}\left(3^{m}\right)$. We have $\theta^{3^{3}}=\theta$ and $3 \mid m=2 h-2$, this leads to $h \equiv 1(\bmod 3)$ and $\theta^{3^{h}}=\theta^{3}$. Plugging $\theta^{3^{h}}=\theta^{3}$ into (6), we have

$$
\theta^{3}=\frac{\theta^{4}+\theta^{2}-\theta+1}{\theta^{4}-\theta^{3}+\theta^{2}+1}
$$

which leads to $\theta^{8}=1$. It follows from $\theta^{3^{3}-1}=1$ that $\theta^{2}=\theta^{\left(8,3^{3}-1\right)}=1$, a contradiction. This completes the proof of Case 2 .

The answer to Open Problem 1.1 is given in the following theorem.

Theorem 3.4. Let $e=3^{h}+5$, where $2 \leq h \leq m-1$. Let $m$ be even. Then the ternary cyclic code $\mathcal{C}_{(1, e)}$ has parameters $\left[3^{m}-1,3^{m}-1-2 m, 4\right]$ if one of the following conditions is met.

1. $m \equiv 0(\bmod 4), m \geq 4$, and $h=\frac{m}{2}$.

2. $m \equiv 2(\bmod 4), m \geq 6$, and $h=\frac{m+2}{2}$.

Proof. The conclusions follow from Lemma3.1, Lemma3.2 Lemma3.3 and Theorem 2.2

\section{Conclusions}

In this paper, we settled an open problem proposed by Ding and Helleseth in 2013 about a class of optimal ternary cyclic codes. The main technique we used is shown in solving the equation in conditions $\mathrm{C} 2$ and $\mathrm{C} 3$. Assume that $\theta$ is a solution of the target equation, we can obtain $\theta^{3^{h}}=R(\theta)$ after calculation, where $R(\theta)$ is a rational function of $\theta$ with known degree and coefficients. Then we take $3^{h}$-th power of $\theta^{3^{h}}=R(\theta)$, together with the relationship between $m$ and $h$, we can find an solvable equation of $\theta$. We remark that when $h$ is close to $\gamma m$, where $\gamma$ is a rational number, our technique always works. For instance, the following theorem gives other optimal cyclic codes with respect to the Sphere Packing bound. This gives an incomplete answer to Open Problems 7.12-7.15 in [6].

Theorem 4.1. Let $m$ be an odd integer no less than five and $\operatorname{gcd}(m, 3)=1$. Then the ternary cyclic code $C_{(1, e)}$ has parameters $\left[3^{m}-1,3^{m}-1-2 m, 4\right]$ if one of the following conditions is met.

1. $e=3^{h}+5$, where $2 h \equiv \pm 1(\bmod m)$;

2. $e=3^{h}+13$, where $2 h \equiv \pm 1(\bmod m)$;

3. $e=\frac{3^{m-1}}{2}+3^{h}+1$, where $2 h \equiv \pm 1(\bmod m)$ or $3 h \equiv \pm 1(\bmod m)$ or $4 h \equiv \pm 1(\bmod m)$.

\section{References}

[1] C. Carlet, C. Ding, and J. Yuan, Linear codes from highly nonlinear functions and their secret sharing schemes, IEEE Trans. Inform. Theory 51 (6) (2005) 2089-2102.

[2] P. Delsarte, On subfield subcodes of modified Reed-Solomon codes, IEEE Trans. Inform. Theory 21 (5) (1975) $575-576$.

[3] C. Ding and J. Yang, Hamming weights in irreducible cyclic codes, Discrete Mathematics 313 (4) (2013) $434-446$.

[4] C. Ding and S. Ling, A q-polynomial approach to cyclic codes, Finite Fields Appl. 20 (3) (2013) 1-14. 
[5] C. Ding, Cyclic codes from some monomials and trinomials, SIAM J. Discrete Mathematics 27 (4) (2013) 19771994.

[6] C. Ding and T. Helleseth, Optimal ternary cyclic codes from monomials, IEEE Trans. Inform. Theory 59 (9) (2013) 5898-5904.

[7] C. Ding and X. Wang, A coding theory construction of new systematic authentication codes, Theor. Comput. Sci. 330 (1) (2005) 81-99.

[8] C. Ding, Y. Yang, and X. Tang, Optimal sets of frequency hopping sequences from linear cyclic codes, IEEE Trans. Inform. Theory 56 (7) (2010) 3605-3612.

[9] H. Dobbertin, T. Helleseth, P. V. Kumar, and H. Martinsen, Ternary m-sequences with three-valued crosscorrelation function: New decimations of Welch and Niho type, IEEE Trans. Inform. Theory 47 (4) (2001) 14731481.

[10] C. Fan, N. Li, and Z. C. Zhou, A class of optimal ternary cyclic codes and their duals, Finite Fields Appl. 37 (2016) 193-202.

[11] K. Feng and J. Luo, Value distribution of exponential sums from perfect nonlinear functions and their applications, IEEE Trans. Inform. Theory 53 (9) (2007) 3035-3041.

[12] T. Feng, On cyclic codes of length $2^{2^{r}}-1$ with two zeros whose dual codes have three weights, Des. Codes Cryptogr 62 (2012) 253-258.

[13] W. C. Huffman and V. Pless, Fundamentals of Error-Correcting Codes, Cambridge University Press, Cambridge, 2003.

[14] D. J. Katz and P. Langevin, Proof of a conjectured three-valued family of Weil sums of binomials, Acta Arith. 169 (2) (2015) 181-199.

[15] T. Kløve, Codes for Error Detection, World Scientific, 2007.

[16] C. J. Li, Q. Yue, and F. W. Li, Weight distributions of cyclic codes with respect to pairwise coprime order elements, Finite Fields Appl. 28 (2014) 94-114.

[17] C. L. Li, N. Li, T. Helleseth, and C. Ding, The weight distributions of several classes of cyclic codes from APN monomials, IEEE Trans. Inform. Theory 60 (8) (2014) 4710-4721.

[18] F. W. Li, Q. Yue, and C. J. Li, The minimum Hamming distances of irreducible cyclic codes, Finite Fields Appl. 29 (2014) 225-242.

[19] N. Li, C. Li, T. Helleseth, C. Ding, and X.H. Tang, Optimal ternary cyclic codes with minimum distance four and five, Finite Fields Appl. 30 (2014) 100-120.

[20] N. Li, Z. C. Zhou and T. Helleseth, On a conjecture about a class of optimal ternary cyclic codes, Seventh International workshop on Signal Design and its Applications in Communications, 2015, 62-65.

[21] J. H. van Lint, Introduction to Coding Theory, 3rd ed. Springer-Verlag, 1999.

[22] B. Schmidt and C. White, All two-weight irreducible cyclic codes, Finite Fields Appl. 8 (2002) 1-17.

[23] L. S. Wang and G. F. Wu, Several classes of optimal ternary cyclic codes with minimal distance four, Finite Fields Appl. 40 (2016) 126-137.

[24] H. D. Yan, Z. C. Zhou, and X. N. Du, A family of optimal ternary cyclic codes from the Niho-type exponent, Finite Fields Appl. 54 (2018) 101-112.

[25] J. Yang, M. Xiong, C. Ding, and J. Luo, Weight distribution of a class of cyclic codes with arbitrary number of zeros, IEEE Trans.Inform. Theory 59 (9) (2013) 5985-5993.

[26] J. Yuan, C. Carlet, and C. Ding, The weight distribution of a class of linear codes from perfect nonlinear functions, IEEE Trans. Inform. Theory 52 (2) (2006) 712-717.

[27] D. B. Zheng, X. Q. Wang, H. Hu, and X. Zeng, The weight distributions of two classes of $p$-ary cyclic codes, Finite Fields Appl. 29 (2014) 202-242.

[28] X. Zeng, J. Shan, and L. Hu, A triple-error-correcting cyclic code from the Gold and Kasami-Welch APN power functions, Finite Fields Appl. 16 (1) (2012) 70-92.

[29] Z. C. Zhou and C. Ding, A class of three-weight cyclic codes, Finite Fields Appl. 25 (2014) 79-93.

[30] Z. C. Zhou and C. Ding, Seven classes of three-weight cyclic codes, IEEE Trans. Commun. 61 (10) (2013) 4120 4126. 